\title{
Jordan isomorphisms and maps preserving spectra of certain operator products
}

\author{
by \\ Jinchuan Hou (Taiyuan), Chi-Kwong Li (Williamsburg) and \\ Ngai-Ching Wong (Kaohsiung and Hong Kong)
}

\begin{abstract}
Let $\mathcal{A}_{1}, \mathcal{A}_{2}$ be (not necessarily unital or closed) standard operator algebras on locally convex spaces $X_{1}, X_{2}$, respectively. For $k \geq 2$, consider different products $T_{1} * \cdots$ $\cdots * T_{k}$ on elements in $\mathcal{A}_{i}$, which covers the usual product $T_{1} * \cdots * T_{k}=T_{1} \cdots T_{k}$ and the Jordan triple product $T_{1} * T_{2}=T_{2} T_{1} T_{2}$. Let $\Phi: \mathcal{A}_{1} \rightarrow \mathcal{A}_{2}$ be a (not necessarily linear) map satisfying $\sigma\left(\Phi\left(A_{1}\right) * \cdots * \Phi\left(A_{k}\right)\right)=\sigma\left(A_{1} * \cdots * A_{k}\right)$ whenever any one of $A_{i}$ 's has rank at most one. It is shown that if the range of $\Phi$ contains all rank one and rank two operators then $\Phi$ must be a Jordan isomorphism multiplied by a root of unity. Similar results for self-adjoint operators acting on Hilbert spaces are obtained.
\end{abstract}

1. Introduction. Spectrum preserving linear maps between Banach algebras have been extensively studied in connection with Kaplansky's problem concerning the characterization of invertibility preserving linear maps; see [14]. A related question is:

Is it true that between semisimple Banach algebras every spectrum preserving unital surjective linear map is a Jordan homomorphism?

Jafarian and Sourour showed in [13] that the answer is positive for maps between $\mathcal{L}\left(X_{1}\right)$ and $\mathcal{L}\left(X_{2}\right)$, the Banach algebras of bounded linear operators acting on complex Banach spaces $X_{1}, X_{2}$, respectively. There are many other papers concerning this type of linear preservers; for example, see $[1-3,10$, 11, 19-21, 23]. We also mention [4, 6, 14] about invertibility preservers and spectrum compressers between semisimple Banach algebras.

2000 Mathematics Subject Classification: Primary 47B49, 47A12.

Key words and phrases: standard operator algebra, spectral functions, Jordan triple products of operators, skew products of operators, nonlinear preserving problems.

Research of the first author was partially supported by NNSFC and PNSFS of China. Research of the second author was partially supported by USA NSF and HK RCG. Research of the third author was partially supported by Taiwan NSC. This research was done while the first author was visiting the College of William and Mary in the Fall of 2005 supported by the Freeman Foundation. 
Recently, there has been growing interest in the problem of characterizing spectrum preserving maps without the linearity assumption. Of course, nonlinear spectrum preserving transformations can be almost arbitrary. So, some mild additional assumptions are needed. In [17], Molnár considered surjective maps $\Phi: \mathcal{L}\left(X_{1}\right) \rightarrow \mathcal{L}\left(X_{2}\right)$ such that $\Phi(A) \Phi(B)$ and $A B$ always have the same spectrum, and proved that such a map must be a Jordan isomorphism multiplied by \pm 1 . In [12], the authors considered surjective maps $\Phi: \mathcal{L}\left(X_{1}\right) \rightarrow \mathcal{L}\left(X_{2}\right)$ such that $\Phi(B) \Phi(A) \Phi(B)$ and $B A B$ always have the same spectrum, and proved that such a map must be a Jordan isomorphism multiplied by a cubic root of unity. In fact, they obtained more general results concerning $\Phi: \mathcal{A}_{1} \rightarrow \mathcal{A}_{2}$, where $\mathcal{A}_{1}, \mathcal{A}_{2}$ are closed unital standard operator algebras on $X_{1}, X_{2}$, respectively. Moreover, in addition to the usual spectrum $\sigma(X)$, they also characterized preservers of the left spectrum, the right spectrum, the boundary of the spectrum, the full spectrum, the point spectrum, the compression spectrum, the approximate point spectrum, and the surjectivity spectrum of operators, etc.; see [7, 8]. Note that all these different types of spectra reduce to the usual spectrum for finite rank operators.

Instead of considering different types of products separately, the authors in [5] considered a general product $T_{1} * \cdots * T_{k}$ on the algebra $M_{n}$ of $n \times n$ complex matrices, which covers the usual product $T_{1} * \cdots * T_{k}=T_{1} \cdots T_{k}$ and the Jordan triple product $T_{1} * T_{2}=T_{2} T_{1} T_{2}$. They showed that a map $\Phi: M_{n} \rightarrow M_{n}$ satisfying

$$
\sigma\left(\Phi\left(A_{1}\right) * \cdots * \Phi\left(A_{k}\right)\right)=\sigma\left(A_{1} * \cdots * A_{k}\right)
$$

for all $A_{1}, \ldots, A_{k}$ in $M_{n}$ must be a Jordan isomorphism multiplied by a root of unity. Their results do not require that $\Phi$ is surjective.

The purposes of this paper are manifold. First, we develop new techniques in $\mathcal{L}(X)$ including various characterizations of rank one operators to extend the results in $[5,12,17]$ to more general settings. These new techniques will be useful in other problems on $\mathcal{L}(X)$. Second, we refine the existing results by weakening the spectrum preserving properties. This will enhance the understanding of the analytic and algebraic properties of spectrum preserving maps on standard operator algebras.

Our results unify and generalize many known facts. In particular, two consequences of our general result (Theorem 3.2) are given below. Here, we suppose that the range of a map $\Phi: \mathcal{A}_{1} \rightarrow \mathcal{A}_{2}$ between standard operator algebras contains all continuous rank one and rank two operators.

(a) If $\sigma\left(\Phi\left(A_{1}\right) \phi\left(A_{2}\right)\right)=\Phi\left(A_{1} A_{2}\right)$ whenever $A_{1}, A_{2}$ in $\mathcal{A}_{1} \operatorname{satisfy} \operatorname{rank}\left(A_{1} A_{2}\right)$ $\leq 1$, then $\Phi$ is a Jordan isomorphism multiplied by \pm 1 .

(b) If $\sigma\left(\Phi\left(A_{1}\right) \phi\left(A_{2}\right) \Phi\left(A_{1}\right)\right)=\Phi\left(A_{1} A_{2} A_{1}\right)$ whenever $A_{1}, A_{2}$ in $\mathcal{A}_{1}$ satisfy $\operatorname{rank}\left(A_{1} A_{2} A_{1}\right) \leq 1$, then $\Phi$ is a Jordan isomorphism multiplied by a complex number $\mu$ with $\mu^{3}=1$. 
We present a special but typical case of our results in Section 2 and their most general forms in Section 3, and obtain analogous results for self-adjoint operators acting on Hilbert spaces in Section 4.

We would like to thank the referee for the kind comments and advice.

2. Results for special operator products $B^{r} A B^{s}$. Let $X$ be a (complex Hausdorff) locally convex (topological linear) space with dual $X^{\prime}$. The $\sigma\left(X, X^{\prime}\right)$ topology is the weakest (Hausdorff) locally convex topology on $X$ such that each $f$ in $X^{\prime}$ defines a continuous linear functional $x \mapsto f(x)$ on $X$. Similarly, we can define the $\sigma\left(X^{\prime}, X\right)$ topology on $X^{\prime}$. Denote by $\mathcal{L}(X)$ the algebra of all continuous linear operators on $X$, and by $\mathcal{F}(X)$ its subalgebra of all continuous finite rank operators $\sum_{i=1}^{n} f_{i} \otimes x_{i}$. Here $f_{i}$ belongs to $X^{\prime}$ and $x_{i}$ belongs to $X$ for each $i=1, \ldots, n$. The operator $f \otimes x$ on $X$ is defined by sending $y$ to $f(y) x$.

Recall that a standard operator algebra $\mathcal{A}$ on a locally convex space $X$ is a subalgebra of $\mathcal{L}(X)$ containing $\mathcal{F}(X)$. We do not, however, assume that $\mathcal{A}$ contains the identity operator $I_{X}$, or that $\mathcal{A}$ is closed in any topology.

The following simple useful lemma was proved in [12]. For the sake of completeness, we give a short proof. Recall that a rank one idempotent on $X$ has the form $f \otimes x$ with $f(x)=1$.

Lemma 2.1. Let $X$ be a locally convex space, and let $A \in \mathcal{L}(X)$. Then $A=0$ if and only if $f(A x)=0$ for all rank one idempotents $f \otimes x$ on $X$.

Proof. We need only check the "only if" part. For any $f$ in $X^{\prime}$ and $x$ in $X$, if $f(x)=\alpha \neq 0$ then $f\left(\frac{1}{\alpha} x\right)=1$ implies $0=f\left(A\left(\frac{1}{\alpha} x\right)\right)=\frac{1}{\alpha} f(A x)$. Thus $f(A x)=0$. If $f(x)=0$, then choose a $g$ in $X^{\prime}$ such that $g(x)=1$. Let $f_{1}=$ $g+f$ and $f_{2}=g-f$. We have $f_{1}(x)=f_{2}(x)=1$. Thus, by the assumption, $f_{1}(A x)=f_{2}(A x)=0$. It follows that $f(A x)=\frac{1}{2}\left(f_{1}-f_{2}\right)(A x)=0$. Hence $A=0$.

Lemma 2.2. Let $X$ be a locally convex space. Let $(r, s)$ be a pair of nonnegative integers such that $r+s \geq 1$. For any $A, \bar{A}$ in $\mathcal{L}(X)$, we have

$$
A=\bar{A} \quad \text { if and only if } \operatorname{tr}\left(B^{r} A B^{s}\right)=\operatorname{tr}\left(B^{r} \bar{A} B^{s}\right)
$$

for every rank one idempotent operator $B$ on $X$.

Proof. For any rank one idempotent $B=f \otimes x$ on $X$, we observe that

$$
\operatorname{tr}\left(B^{r} A B^{s}\right)=\operatorname{tr}\left((f \otimes x)^{r} A(f \otimes x)^{s}\right)=f(A x) f(x)^{r+s-1}
$$

and

$$
\operatorname{tr}\left(B^{r} \bar{A} B^{s}\right)=\operatorname{tr}\left((f \otimes x)^{r} \bar{A}(f \otimes x)^{s}\right)=f(\bar{A} x) f(x)^{r+s-1} .
$$

So, $f((A-\bar{A}) x)=0$ whenever $f(x)=1$. By Lemma $2.1, A=\bar{A}$. 
Lemma 2.3. Let $A$ be any complex $n \times n$ matrix of rank at least $k$. Then there is a unitary $n \times n$ matrix $U$ such that the leading $k \times k$ submatrix of $U^{*} A U$ is nonsingular.

Proof. Suppose $A=P V$ where $P$ is positive semidefinite and $V$ is unitary. Let $U$ be unitary such that $U^{*} V U=D$ is a diagonal unitary matrix. Then $U^{*} A U=\left(U^{*} P U\right) D$, and the sum of the absolute values of the $k \times k$ principal minors of $U^{*} A U$ is the same as the sum of the $k \times k$ principal minors of $U^{*} P U$, which is the $k$ th elementary symmetric function of the eigenvalues of $P$. This is positive because $A$ has rank at least $k$, and therefore $P$ has at least $k$ positive eigenvalues, counting multiplicity. Now, applying a permutation similarity if needed, we see that the leading $k \times k$ submatrix of $U^{*} A U$ is invertible.

Lemma 2.4. Let $X$ be a locally convex space. Let $(r, s)$ be a pair of nonnegative integers such that $r+s \geq 1$. Then a nonzero $A$ in $\mathcal{L}(X)$ has rank one if and only if $\sigma\left(B^{r} A B^{s}\right)$ has at most two elements including 0 for all $B$ in $\mathcal{L}(X)$ with rank 2 .

Proof. We check the sufficiency only. Suppose $A$ has rank at least 2 . Then there are $x_{1}, x_{2}$ in $X$ such that $\left\{A x_{1}, A x_{2}\right\}$ is linearly independent. Let $P$ be the projection of $X$ onto $\left[x_{1}, x_{2}, A x_{1}, A x_{2}\right]$, which is the linear span of $\left\{x_{1}, x_{2}, A x_{1}, A x_{2}\right\}$ of dimension $n \leq 4$. Then we can think of $A_{11}:=P A P$ as an $n \times n$ matrix of rank at least two. By Lemma 2.3, there is a unitary matrix $U$ on $\left[x_{1}, x_{2}, A x_{1}, A x_{2}\right]$ such that $U^{*} A_{11} U$ has an invertible $2 \times 2$ leading submatrix. Thus, we may choose $\widetilde{x}_{1}, \widetilde{x}_{2} \in\left[x_{1}, x_{2}, A x_{1}, A x_{2}\right]$ and a space decomposition of $X$ into $\left[\widetilde{x}_{1}, \widetilde{x}_{2}\right]$ and its complement so that $A$ has an operator matrix form

$$
\left(\begin{array}{cc}
A_{1} & * \\
* & *
\end{array}\right),
$$

where $A_{1}$ is in upper triangular form with nonzero diagonal entries $a_{1}, a_{2}$. Now, use the same space decomposition to construct $B$ so that $B$ has operator matrix $\operatorname{diag}(1, b) \oplus 0$, where $a_{1}, a_{2} b^{r+s}$ are two distinct nonzero numbers. Then $B^{r} A B^{s}$ has two distinct nonzero eigenvalues $a_{1}$ and $a_{2} b^{r+s}$.

The following is our main result. Here, we do not assume the map $\Phi$ is linear, multiplicative or continuous. But these turn out to be parts of the conclusion. Note also that $\Phi$ might not be surjective, and $\mathcal{A}_{1}, \mathcal{A}_{2}$ might not be closed in any topology or contain the identity operators.

TheOREM 2.5. Let $\mathcal{A}_{1}, \mathcal{A}_{2}$ be standard operator algebras on locally convex spaces $X_{1}, X_{2}$, respectively. Let $(r, s)$ be a pair of nonnegative integers such that $r+s \geq 1$. Suppose the range of a map $\Phi: \mathcal{A}_{1} \rightarrow \mathcal{A}_{2}$ contains all 
continuous operators of rank one and rank two, and

$$
\sigma\left(\Phi(B)^{r} \Phi(A) \Phi(B)^{s}\right)=\sigma\left(B^{r} A B^{s}\right)
$$

whenever at least one of $A, B$ in $\mathcal{A}_{1}$ has rank at most one. Then there is a scalar $\lambda$ such that $\lambda^{r+s+1}=1$ and one of the following cases holds:

(1) there is a $\sigma\left(X_{1}, X_{1}^{\prime}\right)-\sigma\left(X_{2}, X_{2}^{\prime}\right)$ bicontinuous invertible linear operator $T: X_{1} \rightarrow X_{2}$ such that

$$
\Phi(A)=\lambda T A T^{-1}, \quad \forall A \in \mathcal{A}_{1} ;
$$

(2) there is a $\sigma\left(X_{1}^{\prime}, X_{1}\right)-\sigma\left(X_{2}, X_{2}^{\prime}\right)$ bicontinuous invertible linear operator $S: X_{1}^{\prime} \rightarrow X_{2}$ such that

$$
\Phi(A)=\lambda S A^{\prime} S^{-1}, \quad \forall A \in \mathcal{A}_{1} .
$$

Here $A^{\prime}: X_{1}^{\prime} \rightarrow X_{1}^{\prime}$ is the dual map of $A: X_{1} \rightarrow X_{1}$.

Proof. It suffices to consider the case when both $X_{1}, X_{2}$ have dimension at least 2. We divide the proof into several steps.

AsSERTION 1. $\Phi$ is injective, sends 0 to 0 and sends rank one operators to rank one operators.

The condition (2.1) implies that

$$
\sigma\left(\Phi(B)^{r} \Phi(f \otimes x) \Phi(B)^{s}\right)=\sigma\left(B^{r} \cdot f \otimes x \cdot B^{s}\right)=\left\{0, f\left(B^{r+s} x\right)\right\}
$$

for all $B$ in $\mathcal{A}_{1}$. Since $\Phi\left(\mathcal{A}_{1}\right)$ contains all continuous operators of rank two, Lemma 2.4 implies that $\Phi$ sends each rank one operator $f \otimes x$ to an operator of rank at most one; the image has rank exactly one, by considering a $B$ in $\mathcal{A}_{1}$ with $f\left(B^{r+s} x\right)=1$ in the spectrum equality above.

It follows from Lemma 2.2 that $\Phi(0)=0$.

Observe that if $\Phi(f \otimes x)=g \otimes y$, then

$$
\begin{aligned}
g\left(\Phi(B)^{r+s} y\right) & =f\left(B^{r+s} x\right), \\
g(y)^{r+s-1} g(\Phi(B) y) & =f(x)^{r+s-1} f(B x), \quad \forall B \in \mathcal{A}_{1} .
\end{aligned}
$$

Setting $B=f \otimes x$, we have

$$
g(y)^{r+s+1}=f(x)^{r+s+1} .
$$

It follows from Lemma 2.1 and (2.3) that $\Phi$ is injective.

Assertion 2. $\Phi(f \otimes x)$ is linear in $f$ when $x$ is fixed, and also linear in $x$ when $f$ is fixed.

Fix $x$ in $X$. Suppose that for $f_{1}, f_{2}$ in $X_{1}^{\prime}$ and a scalar $\alpha$, there are $g_{1}, g_{2}, g, g^{\prime}$ in $X_{2}^{\prime}$ and $y_{1}, y_{2}, y, y^{\prime}$ in $X_{2}$ such that

$$
\Phi\left(f_{i} \otimes x\right)=g_{i} \otimes y_{i} \quad \text { for } i=1,2,
$$

and

$$
\Phi\left(\left(f_{1}+f_{2}\right) \otimes x\right)=g \otimes y, \quad \Phi\left(\alpha f_{1} \otimes x\right)=g^{\prime} \otimes y^{\prime} .
$$


Then (2.2) ensures that

$$
g^{\prime}\left(\Phi(B)^{r+s} y^{\prime}\right)=\alpha f_{1}\left(B^{r+s} x\right)=\alpha g_{1}\left(\Phi(B)^{r+s} y_{1}\right), \quad \forall B \in \mathcal{A}_{1} .
$$

Since the range of $\Phi$ contains all continuous operators of rank one, by Lemma 2.1 for example we have

$$
g^{\prime} \otimes y^{\prime}=\alpha g_{1} \otimes y_{1}, \quad \text { i.e. } \quad \Phi\left(\alpha f_{1} \otimes x\right)=\alpha \Phi\left(f_{1} \otimes x\right) .
$$

On the other hand, (2.2) also ensures that

$$
\begin{aligned}
g\left(\Phi(B)^{r+s} y\right) & =f_{1}\left(B^{r+s} x\right)+f_{2}\left(B^{r+s} x\right) \\
& =g_{1}\left(\Phi(B)^{r+s} y_{1}\right)+g_{2}\left(\Phi(B)^{r+s} y_{2}\right), \quad \forall B \in \mathcal{A}_{1} .
\end{aligned}
$$

This leads, by Lemma 2.1 again, to

$$
g \otimes y=g_{1} \otimes y_{1}+g_{2} \otimes y_{2} .
$$

In other words,

$$
\Phi\left(\left(f_{1}+f_{2}\right) \otimes x\right)=\Phi\left(f_{1} \otimes x\right)+\Phi\left(f_{2} \otimes x\right), \quad \forall f_{1}, f_{2} \in X^{\prime}, x \in X .
$$

As a result, $\Phi(f \otimes x)$ is linear in $f$ when $x$ is fixed. Similarly, $\Phi(f \otimes x)$ is also linear in $x$ when $f$ is fixed.

By counting ranks, we note that (2.5) ensures either

$$
y_{1}=\alpha_{1} y \text { and } y_{2}=\alpha_{2} y
$$

or

$$
g_{1}=\beta_{1} g \quad \text { and } \quad g_{2}=\beta_{2} g
$$

for some scalars $\alpha_{1}, \alpha_{2}, \beta_{1}, \beta_{2}$.

From now on, we make the following

Assumption. The first case (2.6) happens for a linearly independent pair $f_{1}, f_{2}$ in $X_{1}^{\prime}$ and $x$ in $X_{1}$.

Assertion 3. We can define an injective $\sigma\left(X_{1}^{\prime}, X_{1}\right)-\sigma\left(X_{2}^{\prime}, X_{2}\right)$ continuous linear operator $S_{x}^{\prime}: X_{1}^{\prime} \rightarrow X_{2}^{\prime}$ such that

$$
\Phi(f \otimes x)=S_{x}^{\prime} f \otimes y, \quad \forall f \in X_{1}^{\prime} .
$$

To this end, let $f_{3} \in X_{1}^{\prime} \backslash\{0\}, g_{3} \in X_{2}^{\prime}$ and $y_{3} \in X_{2}$ be such that

$$
\Phi\left(f_{3} \otimes x\right)=g_{3} \otimes y_{3} .
$$

Suppose $y_{3}$ were linearly independent of $y$. By counting ranks in

$$
\Phi\left(\left(f_{i}+f_{3}\right) \otimes x\right)=g_{i} \otimes y_{i}+g_{3} \otimes y_{3}=\alpha_{i} g_{i} \otimes y+g_{3} \otimes y_{3}, \quad i=1,2,
$$

we see that $g_{1}, g_{2}$ are both scalar multiples of $g_{3}$. Hence $\Phi\left(f_{1} \otimes x\right)=g_{1} \otimes y_{1}=$ $\lambda g_{2} \otimes y_{2}=\lambda \Phi\left(f_{2} \otimes x\right)$ for some scalar $\lambda$. This implies $\Phi\left(\left(f_{1}-\lambda f_{2}\right) \otimes x\right)=0$, and thus $f_{1}=\lambda f_{2}$. This contradiction tells us that $y_{3}$ also depends on $y$. 
At this stage, we show that for this fixed $x$ in $X_{1}$, we have a fixed $y$ in $X_{2}$ and a linear operator $S_{x}^{\prime}: X_{1}^{\prime} \rightarrow X_{2}^{\prime}$ such that

$$
\Phi(f \otimes x)=S_{x}^{\prime} f \otimes y, \quad \forall f \in X_{1}^{\prime} .
$$

It follows from (2.2) that $S_{x}^{\prime}$ is injective and $\sigma\left(X_{1}^{\prime}, X_{1}\right)-\sigma\left(X_{2}^{\prime}, X_{2}\right)$ continuous.

Assertion 4. For any $\widehat{x}$ in $X_{1}$, there is a $\widehat{y}$ in $X_{2}$ and an injective $\sigma\left(X_{1}^{\prime}, X_{1}\right)-\sigma\left(X_{2}^{\prime}, X_{2}\right)$ continuous linear operator $S_{\widehat{x}}^{\prime}$ such that

$$
\Phi(f \otimes \widehat{x})=S_{\widehat{x}}^{\prime} f \otimes \widehat{y}, \quad \forall f \in X_{1}^{\prime} .
$$

It suffices to consider those $\widehat{x}$ linearly independent of $x$. Assume that for any linearly independent pair $f_{1}, f_{2}$ in $X_{1}^{\prime}$, there are $\widehat{g}_{1}, \widehat{g}_{2}$ in $X_{2}^{\prime}$ and $y_{1}, y_{2}$ in $X_{2}$ such that

$$
\Phi\left(f_{1} \otimes \widehat{x}\right)=\widehat{g}_{1} \otimes y_{1} \quad \text { and } \quad \Phi\left(f_{2} \otimes \widehat{x}\right)=\widehat{g}_{2} \otimes y_{2} .
$$

Claim. $y_{1}, y_{2}$ are linearly dependent.

If not, by counting ranks in

$$
\Phi\left(\left(f_{1}+f_{2}\right) \otimes \widehat{x}\right)=\widehat{g}_{1} \otimes y_{1}+\widehat{g}_{2} \otimes y_{2},
$$

we see that $\widehat{g}_{1}, \widehat{g}_{2}$ are linearly dependent. Suppose also

$$
\Phi\left(f_{1} \otimes x\right)=g_{1} \otimes y \quad \text { and } \quad \Phi\left(f_{2} \otimes x\right)=g_{2} \otimes y .
$$

Here, $g_{i}=S_{x}^{\prime} f_{i}$ for $i=1,2$.

If $y, y_{1}$ are linearly dependent, then as $y_{2}$ is linearly independent of $y_{1}$, we see that $y, y_{2}$ are linearly independent. By counting ranks in

$$
\Phi\left(f_{2} \otimes(x+\widehat{x})\right)=g_{2} \otimes y+\widehat{g}_{2} \otimes y_{2},
$$

we see that $g_{2}, \widehat{g}_{2}$ are linearly dependent. Since $\widehat{g}_{1}, \widehat{g}_{2}$ are linearly dependent, so are $\widehat{g}_{1}, g_{2}$. Note that $f_{1} \otimes \widehat{x}, f_{2} \otimes x$ are linearly independent. Write $y_{1}=\alpha y$ and $\widehat{g}_{1}=\beta g_{2}$ for some nonzero scalars $\alpha, \beta$. Then

$$
\Phi\left(f_{1} \otimes \widehat{x}\right)=\widehat{g}_{1} \otimes y_{1}=\alpha \beta g_{2} \otimes y=\alpha \beta \Phi\left(f_{2} \otimes x\right) .
$$

By $(2.2)$,

$$
f_{1}\left(B^{r+s} \widehat{x}\right)=\alpha \beta f_{2}\left(B^{r+s} x\right), \quad \forall B \in \mathcal{A}_{1} .
$$

By Lemma 2.1, $f_{1} \otimes \widehat{x}=\alpha \beta f_{2} \otimes x$, a contradiction. Hence $y, y_{1}$ are linearly independent, and similarly for $y, y_{2}$. Counting ranks again, we see that $g_{i}, \widehat{g}_{i}$ are linearly dependent for $i=1,2$. This forces $g_{1}, g_{2}$ to be linearly dependent. It follows from the injectivity of $\Phi$ that $f_{1}, f_{2}$ are linearly dependent, a contradiction.

At this point, we can define an injective linear map $S_{\widehat{x}}^{\prime}: X_{1}^{\prime} \rightarrow X_{2}^{\prime}$ such that

$$
\Phi(f \otimes \widehat{x})=S_{\widehat{x}}^{\prime} f \otimes \widehat{y}, \quad \forall f \in X_{1}^{\prime} .
$$

Here $\widehat{y}$ is a fixed element in $X_{2}$. 
Assertion 5. $S_{x}^{\prime} f, S_{\widehat{x}}^{\prime} f$ are linearly dependent for all $f$ in $X_{1}^{\prime}$.

Suppose not, and there is an $f$ in $X_{1}^{\prime}$ such that $g=S_{x}^{\prime} f, \widehat{g}=S_{\widehat{x}}^{\prime} f$ are linearly independent. By definition,

$$
\Phi(f \otimes x)=g \otimes y \quad \text { and } \quad \Phi(f \otimes \widehat{x})=\widehat{g} \otimes \widehat{y} .
$$

Counting ranks in

$$
\Phi(f \otimes(x+\widehat{x}))=g \otimes y+\widehat{g} \otimes \widehat{y},
$$

we see that $y, \widehat{y}$ are linearly dependent. Choose $f_{1}, f_{2}$ in $X_{1}^{\prime}$ such that

$$
f_{1}(x)=f_{1}(\widehat{x})=f_{2}(x)=1 \quad \text { and } \quad f_{2}(\widehat{x})=0 .
$$

By (2.4),

$$
\left|g_{1}(y)\right|=1 \quad \text { and } \quad \widehat{g}_{2}(\widehat{y})=0 .
$$

Here $g_{1}=S_{x}^{\prime} f_{1}$ and $\widehat{g}_{2}=S_{\widehat{x}}^{\prime} f_{2}$. Now, (2.1) gives

$$
\sigma\left(\left(f_{1} \otimes x\right)^{r}\left(f_{2} \otimes \widehat{x}\right)\left(f_{1} \otimes x\right)^{s}\right)=\sigma\left(\left(g_{1} \otimes y\right)^{r}\left(\widehat{g}_{2} \otimes \widehat{y}\right)\left(g_{1} \otimes y\right)^{s}\right) .
$$

Since $\widehat{y}$ linearly depends on $y$, we have $\widehat{g}_{2}(y)=0$, and thus

$$
1=f_{1}(x)^{r+s-1} f_{1}(\widehat{x}) f_{2}(x)=g_{1}(y)^{r+s-1} g_{1}(\widehat{y}) \widehat{g}_{2}(y)=0,
$$

a contradiction.

Now, we can write

$$
\Phi(f \otimes \widehat{x})=S_{x}^{\prime} f \otimes \widehat{y} .
$$

Assertion 6. $\widehat{y}$ is independent of $f$.

Suppose for any other $\bar{f}$ in $X_{1}^{\prime}$, which is linearly independent of $f$, we have $\Phi(\bar{f} \otimes \widehat{x})=S_{x}^{\prime} \bar{f} \otimes \bar{y}$ for some $\bar{y}$ in $X_{2}$. By counting ranks in

$$
\Phi((f+\bar{f}) \otimes \widehat{x})=S_{x}^{\prime} f \otimes \widehat{y}+S_{x}^{\prime} \bar{f} \otimes \bar{y}
$$

we see that $\widehat{y}$ and $\bar{y}$ are linearly dependent, as $S_{x}^{\prime} f$ and $S_{x}^{\prime} \bar{f}$ are independent. Therefore, $\Phi(\bar{f} \otimes \widehat{x})=\alpha S_{x}^{\prime} \bar{f} \otimes \widehat{y}$ for some scalar $\alpha$. On the other hand, $\Phi((f-\bar{f}) \otimes \widehat{x})=\beta S_{x}^{\prime}(f-\bar{f}) \otimes \widehat{y}$ for another scalar $\beta$. This gives $\beta S_{x}^{\prime}(f-\bar{f})=$ $S_{x}^{\prime} f-\alpha S_{x}^{\prime} \bar{f}$, and hence $\alpha=\beta=1$ due to the linear independence of $f$ and $\bar{f}$.

Thus, we can obtain an injective $\sigma\left(X_{1}^{\prime}, X_{1}\right)-\sigma\left(X_{2}^{\prime}, X_{2}\right)$ continuous linear map $S^{\prime}: X_{1}^{\prime} \rightarrow X_{2}^{\prime}$ such that $S^{\prime}=S_{x}^{\prime}$ for all $x$ in $X_{1}$. It then also follows that there is an injective $\sigma\left(X_{1}, X_{1}^{\prime}\right)-\sigma\left(X_{2}, X_{2}^{\prime}\right)$ continuous linear map $T$ : $X_{1} \rightarrow X_{2}$ such that

$$
\Phi(f \otimes x)=S^{\prime} f \otimes T x, \quad \forall f \in X_{1}^{\prime}, x \in X_{1} .
$$

Assertion 7. $S^{\prime}=\lambda T^{-1}$ for some scalar $\lambda$ with $\lambda^{r+s+1}=1$. Here $T^{\prime}: X_{2}^{\prime} \rightarrow X_{1}^{\prime}$ is the dual map of $T$.

We check first that $S^{\prime}$ has dense range. Suppose that $S^{\prime} X_{1}^{\prime}$ is not $\sigma\left(X_{2}^{\prime}, X_{2}\right)$ dense in $X_{2}^{\prime}$. Then there is a nonzero $y$ in $X_{2}$ such that $S^{\prime} f(y)=0$. Since 
the range of $\Phi$ contains all continuous rank one operators on $X_{2}$, there is a $B$ in $\mathcal{A}_{1}$ with $B \neq 0$ and $\Phi(B) X_{2}$ is spanned by $y$. Now by (2.3), we have

$$
f(x)^{r+s-1} f(B x)=S^{\prime} f(T x)^{r+s-1} S^{\prime} f(\Phi(B) T x)=0, \quad \forall f \in X_{1}^{\prime}, \forall x \in X_{1} .
$$

By Lemma 2.1, we have $B=0$. This conflict tells us that $S^{\prime}$ does have dense range in $X_{2}^{\prime}$. Similarly, we see that $T$ has dense range in $X_{2}$. In particular, its dual map $T^{\prime}: X_{2}^{\prime} \rightarrow X_{1}^{\prime}$ is injective.

Applying (2.4), we have

$$
\left(S^{\prime} f(T x)\right)^{r+s+1}=(f(x))^{r+s+1}, \quad \forall f \in X_{1}^{\prime}, x \in X_{1} .
$$

By a connectedness argument, we can derive the existence of a scalar $\lambda$ with $\lambda^{r+s+1}=1$ such that

$$
S^{\prime} f(T x)=\lambda f(x), \quad \forall f \in X_{1}^{\prime}, x \in X_{1} .
$$

It then follows that

$$
T^{\prime} S^{\prime} f=\lambda f, \quad \forall f \in X_{1}^{\prime} .
$$

Since $T^{\prime}$ is now known to be bijective, $S^{\prime}=\lambda T^{\prime-1}$.

At this point, we have shown that

$$
\Phi(A)=\lambda T A T^{-1}, \quad \forall A \in \mathcal{F}\left(X_{1}\right) .
$$

In general, for any $A$ in $\mathcal{A}_{1}, f$ in $X_{1}^{\prime}$ and $x$ in $X_{1}$ with $f(x)=1$, by putting $B=f \otimes x$ in $(2.1)$ we have

$$
f(A x)=\lambda^{r+s}\left(T^{-1}\right)^{\prime} f(\Phi(A) T x)=\lambda^{-1} f\left(T^{-1} \Phi(A) T x\right) .
$$

By Lemma 2.1, we have

$$
\Phi(A)=\lambda T A T^{-1}, \quad \forall A \in \mathcal{A}_{1} .
$$

Finally, if the second case (2.7) happens for all pairs $f_{1}, f_{2}$ in $X_{1}^{\prime}$ and for all $x$ in $X_{1}$, then following a similar, and slightly easier, pattern we will arrive at the other possible conclusions.

Recall that the Mackey topology of a locally convex space $X$ is the (locally convex) topology $\tau\left(X, X^{\prime}\right)$ of uniform convergence on $\sigma\left(X^{\prime}, X\right)$ compact convex subsets of $X^{\prime}$. A locally convex space $X$ is called a Mackey space if its topology coincides with $\tau\left(X, X^{\prime}\right)$. Hilbert spaces, Banach spaces, Fréchet spaces, infrabarrelled spaces, bornological spaces, and Montel spaces are all Mackey spaces.

On the other hand, the strong topology of the dual space $X^{\prime}$ of $X$ is the topology $\beta\left(X^{\prime}, X\right)$ of uniform convergence on bounded subsets of $X$. Equip $X^{\prime}$ with $\beta\left(X^{\prime}, X\right)$ to get the strong dual $X_{\beta}^{\prime}$ of $X$. The strong dual $X_{\beta \beta}^{\prime \prime}$ of $X_{\beta}^{\prime}$ is called the strong bidual of $X$, and $X$ is semireflexive if $K_{X} X=X_{\beta \beta}^{\prime \prime}$, where $K_{X}$ is the canonical embedding of $X$ into $X_{\beta \beta}^{\prime \prime}$. If, in addition, the topology of $X$ agrees with the strong topology then $X$ is reflexive. The MackeyArens theorem implies that $X$ is semireflexive if and only if $\beta\left(X^{\prime}, X\right)=$ 
$\tau\left(X^{\prime}, X\right)$ (see, e.g., [22, Corollary 18.2]). Semireflexive metrizable locally convex spaces are reflexive (see, e.g., [22, Corollary 18.4]). Reflexive locally convex spaces are Mackey spaces.

Recall also that a locally convex space $X$ is barrelled if every $\sigma\left(X^{\prime}, X\right)$ bounded set in $X^{\prime}$ is equicontinuous, and thus relatively $\sigma\left(X^{\prime}, X\right)$ compact by the Alaoglu-Bourbaki theorem (see, e.g., [22, Theorem 16.13]). In other words, $X$ is barrelled if and only if its topology agrees with $\beta\left(X, X^{\prime}\right)$, where we observe $\left(X^{\prime}, \sigma\left(X^{\prime}, X\right)\right)^{\prime}=X$. Banach and Fréchet spaces are barrelled, and barrelled spaces are Mackey.

THEOREM 2.6. In the conclusion of Theorem 2.5, the continuity of $T$ and $S$ can be assumed in the Mackey topology.

(1) Assume Case (1) of Theorem 2.5 occurs. If $X_{1}, X_{2}$ are Banach or Fréchet spaces, then $T$ is a linear homeomorphism in the metric topology.

(2) Assume Case (2) occurs. If $X_{1}$ (resp. $\left.X_{2}\right)$ is barrelled (in particular, Banach or Fréchet), then $X_{2}$ (resp. $\left.X_{1}\right)$ is semireflexive and $X_{1}=$ $\left(X_{2}^{\prime}\right)_{\beta}$ (resp. $\left.X_{2}=\left(X_{1}^{\prime}\right)_{\beta}\right)$. In particular, if both $X_{1}, X_{2}$ are Banach or Fréchet spaces then they are reflexive and dual to each other.

Proof. It is well-known that $\sigma\left(X, X^{\prime}\right)-\sigma\left(Y, Y^{\prime}\right)$ continuous linear operators between $X$ and $Y$ are exactly $\tau\left(X, X^{\prime}\right)-\tau\left(Y, Y^{\prime}\right)$ continuous linear operators. Moreover, if $A$ is a continuous linear operator from a locally convex space $X$ into another $Y$ then $A$ is $\sigma\left(X, X^{\prime}\right)-\sigma\left(Y, Y^{\prime}\right)$ continuous, and its dual map $A^{\prime}: Y^{\prime} \rightarrow X^{\prime}$ is $\beta\left(Y^{\prime}, Y\right)-\beta\left(X^{\prime}, X\right)$ continuous (see, e.g., [22, Proposition 17.14]). Thus $T$ and $S$ in Theorem 2.5 are both continuous in the Mackey topologies. Rather than noting that Banach and Fréchet spaces are Mackey, we can also prove (1) directly by using the closed graph theorem.

For (2), we note that a locally convex space $X$ is semireflexive if and only if $\left(X, \sigma\left(X, X^{\prime}\right)\right)$ is quasi-complete, i.e. all bounded Cauchy nets converges (see, e.g., [22, Theorem 18.2]). Now, $S:\left(X_{1}^{\prime}, \sigma\left(X_{1}^{\prime}, X_{1}\right)\right) \rightarrow\left(X_{2}, \sigma\left(X_{2}, X_{2}^{\prime}\right)\right)$ is a linear homeomorphism. If $X_{1}$ is barrelled, then $\left(X_{1}^{\prime}, \sigma\left(X_{1}^{\prime}, X_{1}\right)\right)$ is quasicomplete, and thus $X_{2}$ is semireflexive. As $S^{\prime}$ induces a linear homeomorphism from $\left(X_{2}^{\prime}, \beta\left(X_{2}^{\prime}, X_{2}\right)\right)$ onto $\left(X_{1}, \beta\left(X_{1}, X_{1}^{\prime}\right)\right)$, we see that $\left(X_{2}^{\prime}\right)_{\beta}=X_{1}$.

On the other hand, the range $\Phi\left(\mathcal{A}_{1}\right)$ is again a standard operator algebra on $X_{2}$. The inverse map $\Phi^{-1}: \Phi\left(\mathcal{A}_{1}\right) \rightarrow \mathcal{A}_{1}$ satisfies a condition similar to (2.1), and clearly the range of $\Phi^{-1}$ contains $\mathcal{F}_{2}\left(X_{1}\right)$. Hence, one can conclude Case 2 again. In case $X_{2}$ is barrelled, we can conclude that $X_{1}$ is semireflexive and $\left(X_{1}^{\prime}\right)_{\beta}=X_{2}$ in a similar manner.

We remark that the barrelledness condition in Theorem 2.6(2) is sharp, as it is known that a Mackey space $X$ has a quasi-complete dual space $\left(X^{\prime}, \sigma\left(X^{\prime}, X\right)\right)$ if and only if $X$ is barrelled (see, e.g., $\left.[15,23.6(4)]\right)$. 
One reason why we are interested in such a generality of Theorem 2.5 is that the whole theory depends on the dual pairs $\left\langle X_{1}, X_{1}^{\prime}\right\rangle,\left\langle X_{2}, X_{2}^{\prime}\right\rangle$ rather than the particular topologies of the underlying spaces $X_{1}, X_{2}$. The following example provides another reason. We think this is a very important case we should not forget.

Example 2.7. Let $X$ be a Banach space. Consider the map $\Phi: \mathcal{L}(X) \rightarrow$ $\mathcal{L}\left(X^{\prime}\right)$ defined by

$$
\Phi(A)=A^{\prime}, \quad \forall A \in \mathcal{L}(X) .
$$

Here $A^{\prime}: X^{\prime} \rightarrow X^{\prime}$ is the dual map of $A$. Note that the range of $\Phi$ might not contain all norm continuous rank one operators on $X^{\prime}$. However, if we equip $X^{\prime}$ with the $\sigma\left(X^{\prime}, X\right)$ topology, then the range of $\Phi$ does contain all $\sigma\left(X^{\prime}, X\right)$ continuous finite rank operators. Thus we can apply Theorem 2.5. Note also that $X$ need not be reflexive. Anyway, we have

$$
\left(X^{\prime}, \sigma\left(X^{\prime}, X\right)\right)_{\beta}^{\prime}=X .
$$

Remark 2.8. We do not have $r=s$ in general, even if Case (2) in Theorem 2.5 holds. Indeed, we always have

$$
\sigma(A B) \cup\{0\}=\sigma(B A) \cup\{0\},
$$

and thus

$$
\begin{aligned}
\sigma\left(B^{r} A B^{s}\right) \cup\{0\} & =\sigma\left(A B^{r+s}\right) \cup\{0\}=\sigma\left(B^{s} A B^{r}\right) \cup\{0\} \\
& =\sigma\left(B^{\prime r} A^{\prime} B^{\prime s}\right) \cup\{0\}=\sigma\left(\Phi(B)^{r} \Phi(A) \Phi(B)^{s}\right) \cup\{0\}
\end{aligned}
$$

for all $A, B$ in $\mathcal{A}_{1}$. We can drop 0 from the above equalities, since $\Phi$ sends invertible elements to invertible elements.

\section{Applications to generalized operator products}

Definition 3.1. Fix a positive integer $k \geq 2$ and a finite sequence $\left(i_{1}, \ldots, i_{m}\right)$ such that $\left\{i_{1}, \ldots, i_{m}\right\}=\{1, \ldots, k\}$ and there is an $i_{p}$ not equal to $i_{q}$ for all other $q$. Define a product for operators $T_{1}, \ldots, T_{k}$ by

$$
T_{1} * \cdots * T_{k}=T_{i_{1}} \cdots T_{i_{m}} .
$$

Clearly, this general product covers the usual product $T_{1} * \cdots * T_{k}=$ $T_{1} \cdots T_{k}$ and the Jordan triple product $T_{1} * T_{2}=T_{2} T_{1} T_{2}$.

Theorem 3.2. Let $\mathcal{A}_{i}$ be a standard operator algebra on a complex locally convex space $X_{i}$ for $i=1,2$. Consider the product of operators $T_{1} * \cdots * T_{k}$ defined in Definition 3.1. Suppose a map $\Phi: \mathcal{A}_{1} \rightarrow \mathcal{A}_{2}$ satisfies

$$
\sigma\left(\Phi\left(A_{1}\right) * \cdots * \Phi\left(A_{k}\right)\right)=\sigma\left(A_{1} * \cdots * A_{k}\right)
$$


whenever any one of $A_{1}, \ldots, A_{k}$ in $\mathcal{A}_{1}$ has rank at most one. Suppose also that the range of $\Phi$ contains all continuous linear operators on $X_{2}$ of rank one and rank two. Then there exists a scalar $\lambda$ such that $\lambda^{m}=1$ and one of the following cases holds.

(1) There exists an invertible operator $T$ in $\mathcal{L}\left(X_{1}, X_{2}\right)$ such that

$$
\Phi(A)=\lambda T A T^{-1} \quad \text { for all } A \in \mathcal{A}_{1} .
$$

(2) There exists an invertible operator $S$ in $\mathcal{L}\left(X_{1}^{\prime}, X_{2}\right)$ such that

$$
\Phi(A)=\lambda S A^{\prime} S^{-1} \quad \text { for all } A \in \mathcal{A}_{1} .
$$

In this case, we have equality of the ordered indices

$$
\left(i_{p+1}, \ldots, i_{m}, i_{1}, \ldots, i_{p-1}\right)=\left(i_{p-1}, \ldots, i_{1}, i_{m}, \ldots, i_{p+1}\right) .
$$

The continuity of $T$ and $S$ above can also be assumed in the Mackey topologies.

Suppose further that $X_{1}$ and $X_{2}$ are Banach or Fréchet spaces. Then T and $S$ are continuous in the metric topologies. If the second case happens, then both $X_{1}$ and $X_{2}$ are reflexive and dual to each other.

Proof. Let $i_{p}$ be a fixed index differing from all other indices $i_{q}$ as in Definition 3.1. We consider only a special class of products $A_{1} * \cdots * A_{k}$ in which $A_{i_{p}}=A$ and all other $A_{i_{q}}=B$ such that one of $A, B$ is of rank zero or one. The condition (3.1) now reduces to the condition (2.1) in Theorem 2.5. Applying Theorems 2.5 and 2.6, we have the desired forms of $\Phi$.

Finally, assume $\Phi(A)=\lambda S A^{\prime} S^{-1}$ as in Case (2). It follows from an argument similar to the one in Remark 2.8 that

$$
\sigma\left(A_{i_{p}} A_{i_{p+1}} \cdots A_{i_{m}} A_{i_{1}} \cdots A_{i_{p-1}}\right)=\sigma\left(A_{i_{p}} A_{i_{p-1}} \cdots A_{i_{1}} A_{i_{m}} \cdots A_{i_{p+1}}\right),
$$

whenever $A_{1}, \ldots, A_{k} \in \mathcal{A}_{1}$ and $A_{i_{p}}=f \otimes x$ has rank one. This amounts to

$$
f\left(A_{i_{p+1}} \cdots A_{i_{m}} A_{i_{1}} \cdots A_{i_{p-1}} x\right)=f\left(A_{i_{p-1}} \cdots A_{i_{1}} A_{i_{m}} \cdots A_{i_{p+1}} x\right)
$$

for all $f$ in $X_{1}^{\prime}$ and $x$ in $X_{1}$. Therefore,

$$
A_{i_{p+1}} \cdots A_{i_{m}} A_{i_{1}} \cdots A_{i_{p-1}}=A_{i_{p-1}} \cdots A_{i_{1}} A_{i_{m}} \cdots A_{i_{p+1}} .
$$

Suppose $i_{p+1} \neq i_{p-1}$. Then we can choose two linearly independent vectors $x_{1}, x_{2}$ in $X_{1}$ and an $f$ in $X_{1}^{\prime}$ such that $f\left(x_{1}\right)=f\left(x_{2}\right)=1$. Set $A_{i_{p+1}}=f \otimes x_{1}$, $A_{i_{p-1}}=f \otimes x_{2}$ and all other $A_{k}$ to be the two-dimensional projection of $X_{1}$ onto the linear span $\left[x_{1}, x_{2}\right]$. In this way, we shall arrive a contradiction

$$
x_{1}=A_{i_{p+1}} \cdots A_{i_{m}} A_{i_{1}} \cdots A_{i_{p-1}} x_{1}=A_{i_{p-1}} \cdots A_{i_{1}} A_{i_{m}} \cdots A_{i_{p+1}} x_{1}=x_{2} .
$$

Therefore, $i_{p+1}=i_{p-1}$. Inductively, we will have the equalities of other indices. 
REMARK 3.3. (a) As mentioned in [5] the assumption that there exists an $i_{p}$ such that $i_{q} \neq i_{p}$ for all other $i_{q}$ in Definition 3.1 is necessary for the conclusion of Theorem 3.2. For example, if $A * B=A A B B$, then one can pick an involution $A_{0}$ different from $I$, and consider $\Phi$ such that $\Phi\left(A_{0}\right)=I$, $\Phi(I)=A_{0}$ and $\Phi(A)=A$ for all other $A$. Then $\Phi$ is surjective such that $\Phi(A) * \Phi(B)$ and $A * B$ always have the same spectrum, but $\Phi$ is not of the form (1) or (2) in Theorem 3.2.

(b) The assumption that the range of $\Phi$ contains all rank two continuous linear operators is necessary for the infinite-dimensional case even if we assume that $\Phi$ is linear and preserves rank one idempotents. For example, let $H$ be an infinite-dimensional complex Hilbert space and $V$ an isometry on $H$ that is not unitary. Let $\Phi: \mathcal{L}(H) \rightarrow \mathcal{L}(H)$ be a linear map defined by $\Phi(A)=V A V^{*}$ for every $A$. Then

$$
\Phi\left(A_{1}\right) * \cdots * \Phi\left(A_{k}\right)=V A_{1} V^{*} * \cdots * V A_{k} V^{*}=V\left(A_{1} * \cdots * A_{k}\right) V^{*}
$$

and

$$
\begin{aligned}
\sigma\left(\Phi\left(A_{1}\right) * \cdots\right. & \left.* \Phi\left(A_{k}\right)\right) \cup\{0\}=\sigma\left(V\left(A_{1} * \cdots * A_{k}\right) V^{*}\right) \cup\{0\} \\
& =\sigma\left(\left(A_{1} * \cdots * A_{k}\right) V^{*} V\right) \cup\{0\}=\sigma\left(A_{1} * \cdots * A_{k}\right) \cup\{0\} .
\end{aligned}
$$

As a result, $\sigma\left(\Phi\left(A_{1}\right) * \cdots * \Phi\left(A_{k}\right)\right)=\sigma\left(A_{1} * \cdots * A_{k}\right)$ whenever $A_{1} * \cdots * A_{k}$ is not invertible. In particular, the equality holds whenever $A_{1} * \cdots * A_{k}$ has finite rank.

Note that, however, Theorems 2.5 and 3.2 indeed apply if we think of $\Phi$ as a map from $\mathcal{L}(H)$ onto $\mathcal{L}(K)$ with $K=V H$.

(c) If Case (1) in Theorem 3.2 holds, then clearly equation (3.1) holds for any operators $A_{1}, \ldots, A_{k}$ in $\mathcal{A}_{1}$. In fact, if the conclusion (1) holds, then $A_{1} * \cdots * A_{k}$ and $\Phi\left(A_{1}\right) * \cdots * \Phi\left(A_{k}\right)$ will always have the same left spectrum, the right spectrum, the boundary of the spectrum, the full spectrum, the point spectrum, the compression spectrum, the approximate point spectrum and the surjectivity spectrum, etc.

(d) Assume Case (2) in Theorem 3.2 holds. In the finite-dimensional case, equation (3.1) will also hold for any matrices $A_{1}, \ldots, A_{k}$. In the infinitedimensional case, one may consider different types of spectra and the same conclusion holds in some occasions, but not always; see [12]. For example, let $X$ be a reflexive infinite-dimensional complex Banach space on which there exists a left invertible operator $A_{0}$ that is not invertible. Thus, $\sigma_{l}\left(A_{0}\right) \neq$ $\sigma_{l}\left(A_{0}^{\prime}\right)$, where $\sigma_{l}(T)$ denotes the left spectrum. Let $\Phi: \mathcal{L}(X) \rightarrow \mathcal{L}(X)$ be a surjective map. Then $\Phi$ satisfies $\sigma_{l}(\Phi(B) \Phi(A) \Phi(B))=\sigma_{l}(B A B)$ for all $A, B$ in $\mathcal{L}(X)$ if and only if there exists an invertible $T$ in $\mathcal{L}(X)$ such that $\Phi(A)=\mu T A T^{-1}$ for all $A$, where $\mu$ is a cubic root of unity. In fact, $\Phi$ satisfies equation (3.1). So, by Theorem 3.2 with $A_{1} * A_{2}=A_{2} A_{1} A_{2}, \Phi$ has either 
the form (1) $\Phi(A)=\mu T A T^{-1}$ for all $A$ or the form (2) $\Phi(A)=\mu S A^{*} S^{-1}$ for all $A$, where $\mu^{3}=1$. However, (2) cannot occur in this situation; otherwise, $\sigma_{l}\left(A_{0}\right)=\sigma_{l}\left(\Phi(I) \Phi\left(A_{0}\right) \Phi(I)\right)=\sigma_{l}\left(A_{0}^{\prime}\right)$.

4. Results on self-adjoint operators. Let $H$ be a complex Hilbert space and $\mathcal{S}(H)$ be the real linear space of all self-adjoint operators in $\mathcal{L}(H)$. Note that $\mathcal{S}(H)$ is a Jordan ring. In this section we solve the problems discussed in Sections 2 and 3 for maps on $\mathcal{S}(H)$. Our results refine those in [5] under the assumption that the range of $\Phi$ contains all self-adjoint operators of rank one and rank two.

It suffices to consider the case when both $X_{1}, X_{2}$ have dimension at least 2 . We begin with an observation.

Lemma 4.1. Let $T_{1} * \cdots * T_{k}=T_{i_{1}} \cdots T_{i_{p}} \cdots T_{i_{m}}$ be a general product on $\mathcal{S}(H)$ defined as in Definition 3.1. Then there exists a positive integer $n$ with

$$
m=2 n-1, \quad i_{p}=n, \quad \text { and } \quad i_{k}=i_{2 n-k} \quad \text { for all } k=1, \ldots, n .
$$

Proof. Since the products are all self-adjoint, we have

$$
T_{i_{1}} \cdots T_{i_{p}} \cdots T_{i_{m}}=T_{i_{m}} \cdots T_{i_{p}} \cdots T_{i_{1}} .
$$

If $i_{1} \neq i_{m}$, we put $T_{i_{1}}=P, T_{i_{m}}=Q$, and all other $T_{i_{j}}=I_{H}$, the identity operator on $H$. Here $P, Q$ are any pair of projections on $H$. Then we get

$$
P Q P \cdots Q=Q P Q \cdots P .
$$

This cannot happen when, e.g., $P=e_{1} \times e_{1}$ and $Q=\frac{1}{\sqrt{2}}\left(e_{1}+e_{2}\right) \times\left(e_{1}+e_{2}\right)$. Here $e_{1}, e_{2}$ are two orthogonal elements of norm one, and the operator $e \times e$ is defined by $x \mapsto\langle x, e\rangle e$. This contradiction shows that $i_{1}=i_{m}$. Inductively, we have $i_{k}=i_{m-k+1}$ for $k=1, \ldots, m$. Since $i_{p}$ is distinct from any other index, we must have $m=2 n-1$ and $i_{p}=n$ for some positive integer $n$.

THEOREM 4.2. Let $\mathcal{S}\left(H_{i}\right)$ be the set of all self-adjoint operators on a complex Hilbert space $H_{i}$ for $i=1,2$. Consider the product $T_{1} * \cdots * T_{k}$ defined in Definition 3.1. Suppose $\Phi: \mathcal{S}\left(H_{1}\right) \rightarrow \mathcal{S}\left(H_{2}\right)$ satisfies

$$
\sigma\left(\Phi\left(A_{1}\right) * \cdots * \Phi\left(A_{k}\right)\right)=\sigma\left(A_{1} * \cdots * A_{k}\right)
$$

whenever any one of $A_{1}, \ldots, A_{k}$ in $\mathcal{S}\left(H_{1}\right)$ has rank at most one. Suppose also that the range of $\Phi$ contains all rank one and rank two self-adjoint operators on $H_{2}$. Then there exist a scalar $\xi$ in $\{-1,1\}$ with $\xi^{m}=1$ and a linear or conjugate linear surjective isometry $U: H_{1} \rightarrow H_{2}$ such that

$$
\Phi(A)=\xi U A U^{*}, \quad \forall A \in \mathcal{S}\left(H_{1}\right) .
$$


To prove our result, it is important to characterize rank one operators in terms of the general products of self-adjoint operators. We have the following lemmas.

Lemma 4.3. Let $(r, s)$ be a pair of nonnegative integers such that $r+s \geq 1$. Let $H$ be a complex Hilbert space, and let $0 \neq A \in \mathcal{S}(H)$. Then the following statements are equivalent.

(a) A has rank one.

(b) For any $B$ in $\mathcal{S}(H)$, the spectrum $\sigma\left(B^{r} A B^{s}\right)$ contains at most one nonzero element.

(c) There does not exist $B$ in $\mathcal{S}(H)$ of rank two such that $\sigma\left(B^{r} A B^{s}\right)$ contains two distinct nonzero elements.

Proof. The implications (a) $\Rightarrow(\mathrm{b}) \Rightarrow(\mathrm{c})$ are clear. The proof of $(\mathrm{c}) \Rightarrow(\mathrm{a})$ is similar to that of Lemma 2.4 .

Proof of Theorem 4.2. Observing that Lemmas 2.1 and 2.2 in Section 2 hold when we consider only rank one self-adjoint idempotents $x \times x$ in $\mathcal{L}(H)$, where $x \times x(y):=\langle y, x\rangle x$ and $\|x\|=1$. Together with Lemma 4.3, Assertion 1 in the proof of Theorem 2.5 is valid. In other words, $\Phi$ is injective, sends 0 to 0 and sends rank one self-adjoint operators to rank one self-adjoint operators. More precisely, for all $x$ in $H_{1}$ there is a $u$ in $H_{2}$, unique up to a complex modulus one multiple, such that

$$
\Phi(x \times x)=\xi u \times u,
$$

where $\xi \in\{-1,1\}$. Write $u=T x$. It follows from the spectrum equality that $\|T x\|=\|x\|$ and $\xi^{m}=1$. As in (2.3), we have

$$
\langle B x, x\rangle=\xi\langle\Phi(B) T x, T x\rangle, \quad \forall B \in \mathcal{S}\left(H_{1}\right), x \in H_{1} .
$$

By a connectedness argument, we see that the choice of $\xi= \pm 1$ is uniform for all $x$ in $H_{1}$.

Putting $B=y \times y$ in (4.2), we see that

$$
|\langle x, y\rangle|=|\langle T x, T y\rangle|, \quad \forall x, y \in H_{1} .
$$

By Wigner's theorem (cf. [9]; see also [16, 18]), we can assume $T$ is either a linear or a conjugate linear isometry from $H_{1}$ into $H_{2}$. If the range of $T$ were not dense in $H_{2}$ then we can choose a self-adjoint operator $B$ on $H_{1}$ such that $B \neq 0, \Phi(B)$ has rank one and $\Phi(B) T x=0$ for all $x$ in $H_{1}$. But then (4.2) leads to the contradiction that $B=0$. Consequently, $T$ is a linear or a conjugate linear isometry from $H_{1}$ onto $H_{2}$. Finally, (4.2) gives

$$
\phi(B)=\xi T B T^{*}, \quad \forall B \in \mathcal{S}\left(H_{1}\right) .
$$




\section{References}

[1] B. Aupetit, Spectrum-preserving linear mappings between Banach algebras or Jordan-Banach algebras, J. London Math. Soc. (2) 62 (2000), 917-924.

[2] B. Aupetit and H. du Toit Mouton, Spectrum preserving linear mappings in Banach algebras, Studia Math. 109 (1994), 91-100.

[3] M. Brešar and P. Šemrl, Linear maps preserving the spectral radius, J. Funct. Anal. 142 (1996), 360-368.

[4] —, - Invertibility preserving maps preserve idempotents, Michigan J. Math. 45 (1998), 483-488.

[5] J. T. Chan, C. K. Li and N. S. Sze, Mappings preserving spectra of product of matrices, Proc. Amer. Math. Soc. 135 (2007), 977-986.

[6] M. D. Choi, D. Hadwin, E. Nordgren, H. Radjavi and P. Rosenthal, On positive linear maps preserving invertibility, J. Funct. Anal. 59 (1984), 462-469.

[7] J. L. Cui and J. C. Hou, Additive maps on standard operator algebras preserving parts of the spectrum, J. Math. Anal. Appl. 282 (2003), 266-278.

[8] - - - Linear maps between Banach algebras compressing certain spectral functions, Rocky Mountain J. Math. 34 (2004), 565-584.

[9] M. Györy, A new proof of Wigner's theorem, Rep. Math. Phys. 54 (2004), 159-167.

[10] J. C. Hou, Spectrum-preserving elementary operators on $\mathcal{B}(X)$, Chinese Ann. Math. Ser. B 19 (1998), 511-516.

[11] —, Rank preserving linear maps on $\mathcal{B}(X)$, Sci. China Ser. A 32 (1989), 929-940.

[12] L. Huang and J. C. Hou, Maps preserving spectral functions of operator products, Chinese Ann. Math. Ser. A, in press.

[13] A. A. Jafarian and A. R. Sourour, Spectrum-preserving linear maps, J. Funct. Anal. 66 (1986), 255-261.

[14] I. Kaplansky, Algebraic and Analytic Aspects of Operator Algebras, CBMS Reg. Conf. Ser. Math. 1, Amer. Math. Soc., Providence, 1970.

[15] G. Köthe, Topological Vector Spaces I, Grundlehren Math. Wiss. 107, Springer, Berlin, 1966.

[16] L. Molnár, Transformations on the set of all n-dimensional subspaces of a Hilbert space preserving principal angles, Comm. Math. Phys. 217 (2001), 409-421.

[17] - Some characterizations of the automorphisms of $\mathcal{B}(H)$ and $\mathcal{C}(X)$, Proc. Amer. Math. Soc. 130 (2002), 111-120.

[18] -, Orthogonality preserving transformations on indefinite inner product space: Generalization of Uhlhorn's version of Wigner's theorem, J. Funct. Anal. 194 (2002), 248-262.

[19] P. Šemrl, Two characterizations of automorphisms on $\mathcal{B}(X)$, Studia Math. 105 (1993), 143-149.

[20] A. R. Sourour, Invertibility preserving linear maps on $\mathcal{L}(X)$, Trans. Amer. Math. Soc. 348 (1996), 13-30.

[21] Q. Wang and J. Hou, Point-spectrum preserving elementary operators on $\mathcal{B}(H)$, Proc. Amer. Math. Soc. 126 (1998), 2083-2088.

[22] Y.-C. Wong, Introductory Theory of Topological Vector Spaces, Pure Appl. Math. 167, Dekker, New York, 1992.

[23] X. L. Zhang and J. C. Hou, Positive elementary operators compressing spectrum, Chinese Sci. Bull. 42 (1997), 270-273. 
Department of Mathematics

Taiyuan University of Technology

Taiyuan 030024, P.R. of China

E-mail: houjinchuan@tyut.edu.cn

Department of Applied Mathematics

National Sun Yat-sen University

and National Center for Theoretical Sciences

Kaohsiung 80424, Taiwan

E-mail: wong@math.nsysu.edu.tw

Current address of N. C. Wong:

Department of Mathematics

The Chinese University of Hong Kong

Hong Kong
Department of Mathematics The College of William \& Mary Williamsburg, VA 13185, U.S.A. E-mail: ckli@math.wm.edu

Received April 1, 2007

Revised version August 10, 2007 